\title{
Symmetry and mass degeneration in multi-Higgs-doublet models
}

\author{
K. Olaussen, ${ }^{a}$ P. Osland ${ }^{b}$ and M. Aa. Solberg ${ }^{a}$ \\ ${ }^{a}$ Department of Physics, NTNU, \\ N-7491 Trondheim, Norway \\ ${ }^{b}$ Department of Physics and Technology, University of Bergen, \\ Postboks 7803, N-5020 Bergen, Norway \\ E-mail: kare.olaussen@ntnu.no, per.osland@ift.uib.no, \\ marius@tf.phys.ntnu.no
}

ABSTRACT: We investigate possible symmetry properties of the scalar sector of MultiHiggs-Doublet Models, and, to some extent, the generalization of such models to gauge groups other than $\mathrm{SU}(2)_{L} \times \mathrm{U}(1)_{Y}$. In models with $C$ (charge conjugation) invariance, and where certain quartic terms are not present, the scalar potential is invariant under a group larger than the gauge group, $O(4)$ when the Higgs fields are doublets. If the Higgs fields develop aligned vacuum expectation values, this symmetry will break to an $O(3)$ subgroup, which in general is further broken by loop corrections involving the gauge bosons. Assuming such corrections are small, the physical properties of the Higgs sector will approximately organize into representations of $\mathrm{SO}(3)$. If the vacuum expectation values of the Higgs fields are aligned in the direction of the $C$ even fields, the mass spectra of the charged and $C$ odd sectors will be degenerate. Moreover, if the Higgs fields develop a pair of non-aligned vacuum expectation values, so that the charge conjugation symmetry is spontaneously broken (but not the U(1) electromagnetic gauge invariance), a pair of light charged pseudo-Goldstone bosons will appear.

Keywords: Higgs Physics, Beyond Standard Model, Global Symmetries, Spontaneous Symmetry Breaking 


\section{Contents}

1 Introduction 1

2 The NHDM potential and Lagrangian $\quad 2$

2.1 The most general $C$-invariant NHDM-potential 4

2.2 Symmetries of $\widehat{\boldsymbol{A}}, \widehat{\boldsymbol{B}}, \widehat{\boldsymbol{C}}$ and $\widehat{\boldsymbol{C}}^{\mathbf{2}}$

$\begin{array}{ll}2.3 \text { Symmetries of the NHDM potential } & 7\end{array}$

2.4 Symmetries of the kinetic terms 8

3 Spontaneous symmetry breakdown $\quad 11$

$\begin{array}{lll}3.1 & \text { Mass-squared matrices } & 12\end{array}$

$\begin{array}{lll}3.2 & \text { The Higgs ghosts } & 15\end{array}$

$\begin{array}{lll}3.3 & \text { Non-aligned vacuum expectation values } & 15\end{array}$

4 Concluding remarks $\quad 18$

A $P(k, \mathbb{R})$, the symmetry group of $\widehat{C}^{2} \quad 18$

$\begin{array}{lr}\text { B The map } \rho & 20\end{array}$

\section{Introduction}

Future discoveries at the LHC may reveal a sector of scalar particles that is much richer than that of the Standard Model (SM). Some of the scalars may be responsible for generating the masses of fermions and the electroweak bosons [1-4], whereas others may be responsible for the dark matter [5-11]. Another interesting aspect of these theories is that CP violation is naturally accommodated, including its spontaneous breaking [12].

It is natural to classify such scalars according to their properties under the $\mathrm{SU}(2)$ associated with the electroweak sector of the Standard Model. In order to be compatible with electroweak precision data, one usually considers only $\mathrm{SU}(2)$ doublets and singlets. Even these representations are severely constrained by the data [13, 13-17].

Going beyond one or two doublets [18-20], one immediately has to face models having a large number of parameters. The structure of such potentials has been studied in [21].

Different doublets could be distinguished via their couplings to fermion fields. This idea is exploited in the so-called Model II version of the two-Higgs-doublet model (2HDM) [18], where one doublet couples to up-type fermions, and the other couples to down-type fermions. Another version of this idea is the one considered in ref. [10], where each fermion or each family has its own Higgs field.

We shall here consider instead the case when the different doublets can not be distinguished (since we are not considering couplings to the fermions). An introduction of 
Yukawa couplings would naturally have broken the symmetry among the different doublets $[22,23]$. Thus, we shall here study the symmetries of models with $N$ doublets - it turns out that by setting a certain $\mathrm{SO}(4)$-violating parameter $\lambda^{(3)}$ to zero and assuming vacuum alignment, the spectrum simplifies considerably. In particular, a certain "custodial" $\mathrm{SO}(3)$ symmetry $[24,25]$ leads to a degeneracy between the mass matrix of the $C$ odd (or equivalently, $C P$ odd) and the charged Higgs bosons.

This possibility of a symmetry group of the scalar polynomial which is larger than required by gauge invariance was pointed out by Weinberg $[26,27]$ many years ago. In the theories considered the extra symmetry was assumed to be a symmetry of any quartic (i.e. renormalizable) potential of the scalar sector.

The Standard Model with its single Higgs doublet is an example of a theory were the most general scalar potential has an extra $O(4)$ symmetry not contained in the $\mathrm{SU}(2) \times \mathrm{U}(1)$ gauge symmetry. An extension of the SM with an extra Higgs doublet (as for instance required if we want to introduce supersymmetry) or more, and with $C$ symmetry, also has a quadratic potential which is automatically $O(4)$ symmetric. This is sufficient to enforce $O(4)$ mass relations up to perturbative corrections in the parameter $g^{\prime}$. (Hence these corrections involve the gauge bosons $Z$ and $\gamma$.) For the quartic potential, the extra $O(4)$ symmetry is broken by the parameter(s) $\lambda^{(3)}$, cf. eq. (2.8). Standard renormalizability instructs us to include into the Lagrangian, all terms allowed by the $\mathrm{SU}(2) \times \mathrm{U}(1)$ symmetry, hence it may be inconsistent to leave out terms proportional to $\lambda^{(3)}$. The presence of such parameters will in general lead to an order $\lambda^{(3)}$ tree-level breaking of the additional symmetry. This is in principle not different from having the symmetry broken by loop corrections. It becomes in any case a question of the magnitude of the perturbation.

We present a detailed classification of the possible terms in the potential, discussing charge conjugation and how the familiar custodial $O(4)$ symmetry of the SM potential generalizes to an $O(4)$ (or, more generally, $O(2 k)$ ) symmetry for certain terms of the potential in the NHDM. The maximal $O(2 k)$ symmetric potential turns out to be a more constrained potential than the maximal charge-invariant one. The kinetic terms are in general subject to an independent classification, depending on the $\mathrm{U}(1)$ hypercharge coupling $g^{\prime}$. We identify, in certain situations, a charged pair whose mass vanishes with $g^{\prime}$.

The paper is organized as follows. In section 2 we discuss the general potential and classify the corresponding Lagrangian, including the kinetic terms. In section 3 we discuss spontaneous symmetry breakdown, and in section 4 we conclude. A couple of more matematical discussions are delegated to appendices.

\section{The NHDM potential and Lagrangian}

We define the $N$-Higgs-doublet model, abbreviated NHDM, to be a system of $N$ two-component complex scalar fields $\Phi_{1}, \Phi_{2}, \ldots, \Phi_{N}$, each with the same transformation property under $\mathrm{SU}(2)_{L} \times \mathrm{U}(1)_{Y}$ as the Higgs field of the Standard Model, and with dynamics defined by the Lagrangian density

$$
\mathcal{L}(x)=\sum_{m}\left[D^{\mu} \Phi_{m}(x)\right]^{\dagger}\left[D_{\mu} \Phi_{m}(x)\right]-V\left(\Phi_{1}, \Phi_{2}, \ldots, \Phi_{N}\right),
$$


where $V\left(\Phi_{1}, \Phi_{2}, \ldots, \Phi_{N}\right)$ is a potential that - in its most general form - is given by $(2.5)$ below. The covariant derivative $D^{\mu}$ is defined as

$$
D^{\mu}=\partial^{\mu}+i g T_{i} W_{i}^{\mu}+i g^{\prime} Y B^{\mu},
$$

where $W_{i}^{\mu}$ and $B^{\mu}$ are the $\mathrm{SU}(2)_{L}$ and $\mathrm{U}(1)_{Y}$ gauge fields, respectively, and $T_{i}=\frac{1}{2} \sigma_{i}$ are the generators of $\mathrm{SU}(2)$ with $\sigma_{1}, \sigma_{2}, \sigma_{3}$ the Pauli matrices. Thus, our Higgs fields are labeled by two indices: The row index running from 1 to $N$ is often written out explicitly as above, and an often hidden group index acted on by the gauge group. The latter are acted on by the matrices $T_{i}$ in (2.2) (whose indices are also hidden). When written explicitly we shall use Greek letters from the beginning of the alphabet.

To write the most general gauge-invariant potential in a renormalizable NHDM in a compact way, we introduce a set of linearly independent ${ }^{1}$ hermitian operators invariant under local $\mathrm{SU}(2)_{L} \times \mathrm{U}(1)_{Y}$ transformations (this is a generalization of the approach for the $2 \mathrm{HDM}$ in $[28])$ :

$$
\begin{aligned}
\widehat{A}_{m} & =\Phi_{m}^{\dagger} \Phi_{m} \\
\widehat{B}_{m n} & =\frac{1}{2}\left(\Phi_{m}^{\dagger} \Phi_{n}+\Phi_{n}^{\dagger} \Phi_{m}\right)=\operatorname{Re}\left(\Phi_{m}^{\dagger} \Phi_{n}\right) \equiv \widehat{B}_{a} \\
\widehat{C}_{m n} & =-\frac{i}{2}\left(\Phi_{m}^{\dagger} \Phi_{n}-\Phi_{n}^{\dagger} \Phi_{m}\right)=\operatorname{Im}\left(\Phi_{m}^{\dagger} \Phi_{n}\right) \equiv \widehat{C}_{a}
\end{aligned}
$$

Due to (anti-)symmetry under interchange of $m$ and $n$ we may impose the restriction that $1 \leq m<n \leq N$, and introduce indices $a, b, \ldots$ labeling such pairs. An explicit invertible encoding is

$$
1 \leq a=a(m, n)=m+\frac{1}{2}(n-2)(n-1) \leq \frac{1}{2} N(N-1) \equiv \mathcal{N} .
$$

We let $m(a), n(a)$ denote the inverse of this encoding. We will use the summation convention that repeated indices from the beginning of the alphabet are summed from 1 to $\mathcal{N}$, and repeated indices from the middle of the alphabet are summed from 1 to $N$. The most general potential in the NHDM thus becomes a linear combination of all different quadratic and quartic factors in the $\Phi_{m}\left(\right.$ and $\Phi_{m}^{\dagger}$ ) which can be formed from $\widehat{A}_{m}, \widehat{B}_{a}$ and $\widehat{C}_{a}:{ }^{2}$

$$
\begin{aligned}
V_{\mathrm{g}}\left(\Phi_{1}, \ldots, \Phi_{N}\right)= & \mu_{m}^{(1)} \widehat{A}_{m}+\mu_{a}^{(2)} \widehat{B}_{a}+\mu_{a}^{(3)} \widehat{C}_{a}+\lambda_{m n}^{(1)} \widehat{A}_{m} \widehat{A}_{n}+\lambda_{a b}^{(2)} \widehat{B}_{a} \widehat{B}_{b} \\
& +\lambda_{a b}^{(3)} \widehat{C}_{a} \widehat{C}_{b}+\lambda_{m a}^{(4)} \widehat{A}_{m} \widehat{B}_{a}+\lambda_{m a}^{(5)} \widehat{A}_{m} \widehat{C}_{a}+\lambda_{a b}^{(6)} \widehat{B}_{a} \widehat{C}_{b}
\end{aligned}
$$

where the "g" in $V_{\mathrm{g}}$ denotes "general". To avoid double counting we introduce the restriction $m \leq n$ in the term involving $\lambda_{m n}^{(1)}$, and the restriction $a \leq b$ in the terms involving $\lambda_{a b}^{(2)}$, $\lambda_{a b}^{(3)}$ and $\lambda_{a b}^{(6)}$. We will not consider terms of degree higher than four, because these would destroy the renormalizability of the model [30]. From the hermiticity of the potential $V_{\mathrm{g}}$

\footnotetext{
${ }^{1}$ There are no linear relations between the operators in (2.3). However, they are algebraically dependent when $N \geq 3$, being restricted by $(N-2)^{2}$ polynomial equations of $8^{\prime}$ th order in the fields.

${ }^{2}$ Since $\left(\Phi_{k}^{\dagger} \sigma^{j} \Phi_{\ell}\right)\left(\Phi_{m}^{\dagger} \sigma^{j} \Phi_{n}\right)=-\left(\Phi_{k}^{\dagger} \Phi_{\ell}\right)\left(\Phi_{m}^{\dagger} \Phi_{n}\right)+2\left(\Phi_{k}^{\dagger} \Phi_{n}\right)\left(\Phi_{m}^{\dagger} \Phi_{\ell}\right)$, other quartic invariants may be expressed by those chosen.
} 
all parameters $\mu$ and $\lambda$ in the expansion (2.5) must be real. Thus the number of free real parameters in $(2.5)$ is

$$
N_{\text {tot }}=N+2 \mathcal{N}+\frac{1}{2} N(N+1)+\mathcal{N}(\mathcal{N}+1)+2 N \mathcal{N}+\mathcal{N}^{2}=\frac{1}{2} N^{2}\left(N^{2}+3\right),
$$

which for $N=1$ gives us the 2 parameters of the Standard Model $\left(\mu^{2}\right.$ and $\left.\lambda\right) . N=2$ gives us the usual 14 parameters for the 2HDM. There are 54 parameters for $N=3$ and 152 parameters for $N=4$.

This counting does not take into account the fact that we may make $\mathrm{SU}(N)$ row transformations on the fields $\Phi_{m}$ to eliminate some terms in (2.5). One possible choice is to transform the quadratic terms into a diagonal form, i.e. so that $\mu_{a}^{(2)}=\mu_{a}^{(3)}=0$. This in general leaves a matrix of $N-1$ independent diagonal phase transformations (such that the determinant is unity). We may for instance use it to transform all $\lambda_{1 a}^{(5)}$ with $n(a)=m(a)+1$ to zero. This reduces the number of parameters by $N^{2}-1$, i.e. to $N_{\text {tot }}^{\prime}=\frac{1}{2}\left(N^{4}+N^{2}+2\right)$, yielding 11 parameters for $N=2$ (in agreement with Davidson and Haber [19]), 46 parameters for $N=3$, and 137 parameters for $N=4$.

\subsection{The most general $C$-invariant NHDM-potential}

The charge conjugation operator $C$ is a linear operator, multiplicative in the fields, which leaves complex constants unaltered, but maps fields onto their hermitian conjugate transposed; $C\left(z \Phi_{m}\right)=z \Phi_{m}^{\dagger T}$, where $z$ is a complex number. ${ }^{3}$ Then $C\left(\widehat{C}_{a}\right)=-\widehat{C}_{a}$, in contrast to $C\left(\widehat{A}_{m}\right)=\widehat{A}_{m}$, and $C\left(\widehat{B}_{a}\right)=\widehat{B}_{a}$. We obtain a $C$-invariant potential by leaving out all terms which are odd in $\widehat{C}_{a}$, i.e., terms involving $\mu_{a}^{(3)}, \lambda_{m a}^{(5)}$, and $\lambda_{a b}^{(6)}$. There are $\mathcal{N}+N \mathcal{N}+\mathcal{N}^{2}=\frac{1}{4} N(N-1)\left(N^{2}+N+2\right)$ such terms, leaving

$$
N_{C}=\frac{1}{4} N\left(N^{3}+5 N+2\right)
$$

free parameters for the general renormalizable $C$-invariant potential,

$$
\begin{aligned}
V_{C}\left(\Phi_{1}, \ldots, \Phi_{N}\right)= & \mu_{m}^{(1)} \widehat{A}_{m}+\mu_{a}^{(2)} \widehat{B}_{a}+\lambda_{m n}^{(1)} \widehat{A}_{m} \widehat{A}_{n}+\lambda_{a b}^{(2)} \widehat{B}_{a} \widehat{B}_{b} \\
& +\lambda_{a b}^{(3)} \widehat{C}_{a} \widehat{C}_{b}+\lambda_{m a}^{(4)} \widehat{A}_{m} \widehat{B}_{a} .
\end{aligned}
$$

For $N=1$ we get the usual 2 parameters of the standard model, the Higgs potential of which is automatically $C$-invariant. For $N=2$ we get the usual (see e.g., [28]) 10 parameters. For $N=3$ we get 33 parameters, and for $N=4$ we get 86 parameters.

This counting does not take into account that we may make $O(N)$ transformations on the row of fields $\Phi_{m}$ to eliminate some terms in (2.8). A natural choice is to transform the quadratic terms to diagonal form, i.e. so that $\mu_{a}^{(2)}=0$. This reduces the number of parameters by $\frac{1}{2} N(N-1)$, i.e. to $N_{C}^{\prime}=\frac{1}{4} N\left(N^{3}+3 N+4\right)$. This gives 9 parameters for $N=2$ (in agreement with [20]), 30 parameters for $N=3$, and 80 parameters for $N=4$.

The difference $N_{\text {phases }}=N_{\text {tot }}^{\prime}-N_{C}^{\prime}=\frac{1}{4} N^{2}\left(N^{2}-1\right)+1-N$ counts the number of genuine $C$-violating parameters in $V_{\mathrm{g}}$ (in agreement with Branco et al. [29]).

\footnotetext{
${ }^{3}$ This definition assumes that we for some reasons have decided on a decomposition of all fields into their real and imaginary parts. It is not invariant under complex transformations of the fields, see e.g. [29].
} 


\subsection{Symmetries of $\widehat{A}, \widehat{B}, \widehat{C}$ and $\widehat{C}^{2}$}

For generality we here consider $k$ (rather than 2)-component fields, i.e. with $\mathrm{SU}(k) \times \mathrm{U}(1)$ as gauge group. To make it simpler to explore all possible symmetries we express the field $\Phi_{m}$ in terms of its independent real (hermitian) components, $\Phi_{m}=\Psi_{m}+\mathrm{i} \Theta_{m}$. Define $2 k \times 2 k$ matrices

$$
\mathcal{I}=\left(\begin{array}{cc}
I_{k} & 0_{k} \\
0_{k} & I_{k}
\end{array}\right), \quad \mathcal{J}=\left(\begin{array}{rr}
0_{k} & I_{k} \\
-I_{k} & 0_{k}
\end{array}\right)
$$

where the subscript $k$ indicates the linear dimension of the submatrix involved. The complex scalar product between two fields $\Phi_{m}$ and $\Phi_{n}$, invariant under unitary (U $\left.(k)\right)$ transformations, can be expressed in terms of two real bilinear forms ${ }^{4}$

$$
\begin{aligned}
& \operatorname{Re}\left(\Phi_{m}^{\dagger} \Phi_{n}\right)=\widehat{B}_{m n}=\left(\Psi_{m}^{T}, \Theta_{m}^{T}\right) \mathcal{I}\left(\begin{array}{c}
\Psi_{n} \\
\Theta_{n}
\end{array}\right)=\Psi_{m}^{T} \Psi_{n}+\Theta_{m}^{T} \Theta_{n} \\
& \operatorname{Im}\left(\Phi_{m}^{\dagger} \Phi_{n}\right)=\widehat{C}_{m n}=\left(\Psi_{m}^{T}, \Theta_{m}^{T}\right) \mathcal{J}\left(\begin{array}{c}
\Psi_{n} \\
\Theta_{n}
\end{array}\right)=\Psi_{m}^{T} \Theta_{n}-\Theta_{m}^{T} \Psi_{n} .
\end{aligned}
$$

The first is the Euclidean dot product between $2 k$-component real vectors, the second is the Poisson bracket (symplectic form) of the same quantities viewed as coordinates and momenta of $2 k$-dimensional phase space. The quantities in (2.10) are individually invariant under transformation groups larger than $\mathrm{U}(k)$. The first form $\widehat{B}$ (with $\widehat{A}$ as a special case) is invariant under the $O(2 k)$ group of real orthogonal transformations,

$$
\left(\begin{array}{c}
\Psi_{n} \\
\Theta_{n}
\end{array}\right) \rightarrow O\left(\begin{array}{c}
\Psi_{n} \\
\Theta_{n}
\end{array}\right), \quad O^{T} O=\mathcal{I}
$$

the second form $\widehat{C}$ is invariant under the $\operatorname{Sp}(k, R)$ group of real symplectic transformations,

$$
\left(\begin{array}{c}
\Psi_{n} \\
\Theta_{n}
\end{array}\right) \rightarrow S\left(\begin{array}{c}
\Psi_{n} \\
\Theta_{n}
\end{array}\right), \quad S^{T} \mathcal{J} S=\mathcal{J}
$$

In this formulation the charge conjugation operator $C$ discussed above can be represented as a particular $O(2 k)$ transformation when acting on the fields $\Psi_{n}$ and $\Theta_{n}$

$$
C=\left(\begin{array}{rr}
I_{k} & 0_{k} \\
0_{k} & -I_{k}
\end{array}\right) .
$$

Considering infinitesimal transformations, $O=\mathcal{I}+\epsilon L+\mathcal{O}\left(\epsilon^{2}\right), S=\mathcal{I}+\epsilon M+\mathcal{O}\left(\epsilon^{2}\right)$, the conditions (2.11) and (2.12) become

$$
L^{T} \mathcal{I}+\mathcal{I} L=0_{2 k}, \quad M^{T} \mathcal{J}+\mathcal{J} M=0_{2 k}
$$

\footnotetext{
${ }^{4}\left\langle\Phi_{m}, \Phi_{n}\right\rangle=\Phi_{m}^{*} \cdot \Phi_{n}=\widehat{B}_{m n}+i \widehat{C}_{m n}$
} 
Thus $L$ must be a $2 k \times 2 k$ antisymmetric real matrix; there is a set (Lie algebra) $s o(2 k)$ of $2 k^{2}-k$ linearly independent such matrices. Writing out the condition for $M$ in terms of $k \times k$ submatrices we find that it must have the form

$$
M=\left(\begin{array}{cc}
A & B \\
C & -A^{T}
\end{array}\right), \quad B=B^{T}, C=C^{T} .
$$

There is a set $s p(k)$ of $k^{2}+k(k+1)=2 k^{2}+k$ linearly independent such matrices. The infinitesimal transformations of the original $\mathrm{U}(k)$ are the intersection of the sets $s o(2 k)$ and $s p(k)$. I.e., the matrices of the form $\left(\begin{array}{cc}A & B \\ -B & A\end{array}\right)$, with $A=-A^{T}$ and $B^{T}=B$. There are $\frac{1}{2} k(k-1)+\frac{1}{2} k(k+1)=k^{2}$ such linearly independent matrices.

The symmetries of $\widehat{C}^{2}$. The form $\widehat{C}^{2}$ (or more precisely $\widehat{C}_{m n} \widehat{C}_{m^{\prime} n^{\prime}}$ ) has a bigger symmetry group than the form $\widehat{C}$. Still, we will see that such operators (forms) will violate the full $O(4)$ symmetry we can assign the rest of the Lagrangian. In analogy with (2.12), $\widehat{C}^{2}$ symmetries are given by

$$
\left(\begin{array}{c}
\Psi_{n} \\
\Theta_{n}
\end{array}\right) \rightarrow S\left(\begin{array}{c}
\Psi_{n} \\
\Theta_{n}
\end{array}\right), \quad S^{T} \mathcal{J} S= \pm \mathcal{J}
$$

which can be collected in a set

$$
P(k, \mathbb{R})=\left\{S \in G L_{2 k}(\mathbb{R}) \mid S^{T} \mathcal{J} S= \pm \mathcal{J}\right\},
$$

which we in appendix A show is a Lie group.

The component

$$
P^{-}(k, \mathbb{R})=\left\{S \in G L_{2 k}(\mathbb{R}) \mid S^{T} \mathcal{J} S=-\mathcal{J}\right\},
$$

consists of matrices with determinant

$$
\operatorname{det}\left(P^{-}(k, \mathbb{R})\right)=(-1)^{k}
$$

as shown in appendix A. The group $P(k, \mathbb{R})=\operatorname{Sp}(k, \mathbb{R}) \cup P^{-}(k, \mathbb{R})$ will have the same Lie algebra as $\operatorname{Sp}(k, \mathbb{R})$, since the new component $P^{-}(k, \mathbb{R})$ is not connected with the identity. This is manifested by the equation corresponding to eq. (2.14),

$$
\mathcal{J}+\epsilon\left(M^{T} \mathcal{J}+\mathcal{J} M\right)= \pm \mathcal{J}
$$

not having any solution for the case of a $-\mathcal{J}$ on the right side, see appendix A for a proof.

The most general $\boldsymbol{O}(\mathbf{2 k})$-symmetric potential. We can conclude that the most general $O(2 k)$-invariant potential can be written

$$
\begin{aligned}
V_{O(2 k)}\left(\Phi_{1}, \ldots, \Phi_{N}\right)= & \mu_{m}^{(1)} \widehat{A}_{m}+\mu_{a}^{(2)} \widehat{B}_{a}+\lambda_{m n}^{(1)} \widehat{A}_{m} \widehat{A}_{n} \\
& +\lambda_{a b}^{(2)} \widehat{B}_{a} \widehat{B}_{b}+\lambda_{m a}^{(4)} \widehat{A}_{m} \widehat{B}_{a}
\end{aligned}
$$


since we have seen that operators not containing any factor $\widehat{C}$ are $O(2 k)$-invariant. We obtain an $O(2 k)$-invariant potential by leaving out terms proportional to $\lambda_{a b}^{(3)}$ from the $C$ invariant potential $V_{C}(2.8)$. The number of terms in $V_{O(2 k)}$ is then $N_{O(2 k)}=N_{C}-\mathcal{N}^{2}=$ $\frac{1}{4} N\left(N^{3}+5 N+2\right)-\frac{1}{4} N^{2}(N-1)^{2}$, giving

$$
N_{O(2 k)}=\frac{1}{2} N(N+1)^{2}
$$

free parameters for the general renormalizable $O(2 k)$-invariant potential $V_{O(2 k)}$. For $N=1$ we get the usual 2 parameters of the standard model, the Higgs potential being automatically $O(2 k)$-invariant. For $N=2$ we get the usual 9 parameters, one parameter less than for the $C$-invariant potential. For $N=3$ we get 24 parameters, and for $N=4$ we get 50 parameters.

This counting does again not take into account that we may make $O(N)$ transformations on the row of fields $\Phi_{m}$ to eliminate some terms in (2.21). We may transform the quadratic terms to diagonal form, so that $\mu_{a}^{(2)}=0$. This reduces the number of parameters by $\frac{1}{2} N(N-1)$, i.e. to $N_{O(2 k)}^{\prime}=\frac{1}{2} N\left(N^{2}+N+2\right)$. This gives 8 parameters for $N=2,21$ parameters for $N=3$, and 44 parameters for $N=4$.

\subsection{Symmetries of the NHDM potential}

Since the NHDM-potential $V_{\mathrm{g}}$ is constructed from the invariants (2.3) the symmetries of the latter are reflected in the symmetries of the former, but in a manner depending on details of the construction:

1. If $V_{\mathrm{g}}$ depends only on the $\widehat{A}_{m}$ 's, i.e. if only the parameters $\mu_{m}^{(1)}$ and $\lambda_{m n}^{(1)}$ are nonzero, then the symmetry group of $V_{\mathrm{g}}$ is at least ${ }^{5} \bigotimes_{m=1}^{N} O(2 k)$, since we can make independent transformations on each $\Phi_{m}$.

2. If $V_{\mathrm{g}}$ depends only on the $\widehat{A}_{m}$ 's and the $\widehat{B}_{a}$ 's, i.e. for a $C$-invariant theory $(2.8)$, where in addition the parameters $\lambda_{a b}^{(3)}=0$, then the symmetry group of $V_{\mathrm{g}}$ is at least $O(2 k)$. It may contain several such factors if some of the parameters $\mu_{a}^{(2)}$ and $\lambda_{a b}^{(2)}$ vanish. To analyze this we partition the $\Phi_{m}$ 's into sets: If a parameter $\mu_{a}^{(2)}$ is nonzero, then the fields $\Phi_{m(a)}$ and $\Phi_{n(a)}$ belong to the same set, with $m(a)$ and $n(a)$ denoting that $m$ and $n$ are contained in $a$. If a parameter $\lambda_{a b}^{(2)}$ is nonzero, then the fields $\Phi_{m(a)}$ and $\Phi_{n(a)}$ belong to the same set, and the fields $\Phi_{m(b)}$ and $\Phi_{n(b)}$ belong to the same set. With this partitioning into a maximal number of sets we may make one independent $O(2 k)$ transformation for each set.

3. If $V_{\mathrm{g}}$ depends only on the $\widehat{C}_{a}$ 's, i.e. with only the parameters $\mu_{a}^{(3)}$ and $\lambda_{a b}^{(3)}$ being nonzero, then the symmetry group of $V_{\mathrm{g}}$ is at least $\operatorname{Sp}(k, \mathbb{R})$. If we (in the same manner as above) can partition the fields into several sets, then we may make independent $\operatorname{Sp}(k, \mathbb{R})$ transformations on fields belonging to different sets. However, since the

\footnotetext{
${ }^{5}$ It could possibly be larger, since there might be additional row symmetries transforming fields $\Phi_{m}$ with different $m$ into each other; such symmetries would require special relations among the parameters $\mu_{m}^{(1)}$ and $\lambda_{m n}^{(1)}$.
} 
additional symmetries in this case fail to be symmetries of even the zero'th order kinetic term (2.30), their significance is uncertain.

4. With all parameters arbitrary the symmetry group of $V_{\mathrm{g}}$ is just the original $\mathrm{SU}(k) \times$ $\mathrm{U}(1)$ gauge symmetry.

In this work we will pay special attention to the second scenario, with $k=2$.

\subsection{Symmetries of the kinetic terms}

We now turn to the (global) symmetries of the kinetic terms of the Lagrangian,

$$
K=\sum_{n=1}^{N}\left[\left(\partial^{\mu}+G^{\mu}\right) \Phi_{n}(x)\right]^{\dagger}\left[\left(\partial_{\mu}+G_{\mu}\right) \Phi_{n}(x)\right],
$$

with

$$
G^{\mu}=i g T_{i} W_{i}^{\mu}+i g^{\prime} Y B^{\mu} .
$$

Let $K_{i}$ denote the terms of the $i$ 'th order in the gauge fields.

Consider the transformation of the kinetic terms linear in the gauge fields, $K_{1}$, under the map $\rho$ defined in appendix B. We can then write ${ }^{6}$

$$
\begin{aligned}
K_{1} & =\sum_{n=1}^{N} \partial^{\mu}\left(\Phi_{n}\right)_{k}^{\dagger} G_{\mu}\left(\Phi_{n}\right)_{k}+\left(\Phi_{n}\right)_{k}^{\dagger} G^{\mu \dagger} \partial_{\mu}\left(\Phi_{n}\right)_{k} \\
& =\sum_{n=1}^{N} \rho\left(\partial^{\mu}\left(\Phi_{n}\right)_{k}^{\dagger}\right) \rho\left(G_{\mu}\right) \rho\left(\left(\Phi_{n}\right)_{k}\right)+\rho\left(\left(\Phi_{n}\right)_{k}^{\dagger}\right) \rho\left(G^{\mu \dagger}\right) \rho\left(\partial_{\mu}\left(\Phi_{n}\right)_{k}\right) \\
& =\sum_{n=1}^{N} \partial^{\mu} \Phi_{n}^{T} \mathcal{T}_{\mu} \Phi_{n}+\Phi_{n}^{T}\left(-\mathcal{T}^{\mu}\right) \partial_{\mu} \Phi_{n}
\end{aligned}
$$

where the subscript $k$ in $\left(\Phi_{n}\right)_{k}$ indicates this is the usual complex Higgs $k$-plet (in the case $k=2$ the usual complex Higgs doublet), while $\Phi_{n}$ is the $2 k$ dimensional real vector

$$
\Phi_{n}=\left(\begin{array}{c}
\Psi_{n} \\
\Theta_{n}
\end{array}\right)
$$

where $\left(\Phi_{n}\right)_{k}=\Psi_{n}+i \Theta_{n}$, and where we also use eqs. (B.2), (B.3) and (B.6).

In eq. (2.25) we have applied the transformation $\rho$ on the gauge terms $G^{\mu}$ defined in eq. (2.24),

$$
\rho\left(G^{\mu}\right)=\mathcal{T}^{\mu}, \quad \rho\left(G^{\mu \dagger}\right)=-\mathcal{T}^{\mu},
$$

where $\mathcal{T}$ then reads

$$
\mathcal{T}^{\mu}=\left(\begin{array}{cc}
g W_{I}^{\mu} & -g W_{R}^{\mu}-g^{\prime} Y B^{\mu} \\
g W_{R}^{\mu}+g^{\prime} Y B^{\mu} & g W_{I}^{\mu}
\end{array}\right),
$$

\footnotetext{
${ }^{6}$ Disregarding so-called Schwinger terms - here terms proportional to $i\left[\partial_{\mu} \phi(x), \phi(x)\right]$ for a scalar field $\phi$ - or, alternatively, reasoning classically.
} 
with $W_{R}^{\mu}=\sum_{i}^{\prime} W_{i}^{\mu} T_{i}^{\mathrm{s}}$, summed over the set of real symmetric generators $T_{i}^{s}$ of $\mathrm{SU}(k)$, and $W_{I}^{\mu}=\mathrm{i} \sum_{i}^{\prime} W_{i}^{\mu} T_{i}^{\mathrm{a}}$, summed over the set of imaginary antisymmetric generators $T_{i}^{a}$. For $k=2$ the two sets are respectively $\left\{\frac{1}{2} \sigma^{1}, \frac{1}{2} \sigma^{3}\right\}$ and $\left\{\frac{1}{2} \sigma^{2}\right\}$.

Finally, we consider the kinetic terms quadratic in the gauge fields,

$$
\begin{aligned}
K_{2} & =\sum_{n=1}^{N}\left(\Phi_{n}\right)_{k}^{\dagger} G^{\mu \dagger} G_{\mu}\left(\Phi_{n}\right)_{k} \\
& =\sum_{n=1}^{N} \rho\left(\left(\Phi_{n}\right)_{k}^{\dagger}\right) \rho\left(G^{\mu \dagger}\right) \rho\left(G_{\mu}\right) \rho\left(\left(\Phi_{n}\right)_{k}\right) \\
& =-\sum_{n=1}^{N} \Phi_{n}^{T} \mathcal{T}^{2} \Phi_{n} .
\end{aligned}
$$

The symmetries of $\boldsymbol{K}_{\mathbf{0}}$. When we first ignore couplings to the gauge fields the remaining terms can be written

$$
K_{0}=\sum_{n=1}^{N} \sum_{\alpha=1}^{k}\left(\partial^{\mu} \Psi_{n \alpha} \partial_{\mu} \Psi_{n \alpha}+\partial^{\mu} \Theta_{n \alpha} \partial_{\mu} \Theta_{n \alpha}\right),
$$

where the group index $\alpha$ labels the $k$ components of $\Phi_{n}=\Psi_{n}+\mathrm{i} \Theta_{n}$. This term is invariant under rotation of all components $\left\{\Psi_{n \alpha}, \Theta_{n \alpha}\right\}$ into each other. I.e., the symmetry group of $K_{0}$ is $O(2 k N)$. The connected part of this group is $\mathrm{SO}(2 k N)$, whose generators are all real antisymmetric matrices, $L_{m n, \alpha \beta}=-L_{n m, \beta \alpha}$ (i.e. $L^{T}=-L$, where transposition refers to both sets of indices).

The symmetries of $\boldsymbol{K}_{\mathbf{0}}$ and $\boldsymbol{K}_{\mathbf{1}}$. Next, consider the terms linear in the gauge fields again, cf. eq. (2.25),

$$
K_{1}=\sum_{n=1}^{N}\left(\left(\partial_{\mu}\left(\Psi_{n}^{T}, \Theta_{n}^{T}\right)\right) \mathcal{T}\left(\begin{array}{c}
\Psi_{n} \\
\Theta_{n}
\end{array}\right)-\left(\Psi_{n}^{T}, \Theta_{n}^{T}\right) \mathcal{T}^{\mu} \partial_{\mu}\left(\begin{array}{c}
\Psi_{n} \\
\Theta_{n}
\end{array}\right)\right),
$$

with $\mathcal{T}$ given in eq. (2.28).

Consider now an infinitesimal transformation $\delta \Phi_{m, \alpha}=L_{m n, \alpha \beta} \Phi_{n, \beta}$, and $\mathcal{T}$ denoting the $2 k \times 2 k$ antisymmetric matrix in equation (2.28) (in group indices $\alpha, \beta$ - in addition it is proportional to the $N \times N$ unit matrix in row indices). The requirement that this is an infinitesimal symmetry transformation for $K_{1}$ is that $L^{T} \mathcal{T}+\mathcal{T} L=0$. Or, when we restrict $L$ to be antisymmetric so that it also is an infinitesimal symmetry transformation for $K_{0}$,

$$
L_{m n, \alpha \beta} \mathcal{T}_{\beta \gamma}-\mathcal{T}_{\alpha \beta} L_{m n, \beta \gamma}=0 .
$$

In order to determine the allowed structure of $L$, we expand these matrices into terms of definite symmetries $\left(L^{(\mathrm{s})}\right.$ symmetric, and $L^{(\mathrm{a})}$ antisymmetric) in the $m n$ indices:

$$
L_{m n, \alpha \beta}=\sum\left(S_{\alpha \beta} L_{m n}^{(\mathrm{a})}+A_{\alpha \beta} L_{m n}^{(\mathrm{s})}\right),
$$


with $S$ (symmetric) and $A$ (antisymmetric) restricted by the constraint (2.32). The sum runs over all possible combinations of allowed matrices. ${ }^{7}$ We next note that the antisymmetric matrices $\mathcal{T}$ can be expanded in the set

$$
\widehat{\mathcal{T}}=\left\{T_{i}^{\mathrm{a}} \mathcal{I}, T_{i}^{\mathrm{s}} \mathcal{J}, \mathcal{J}\right\}=\left\{\left(\begin{array}{cc}
T_{i}^{\mathrm{a}} & 0_{k} \\
0_{k} & T_{i}^{\mathrm{a}}
\end{array}\right),\left(\begin{array}{cc}
0_{k} & T_{i}^{\mathrm{s}} \\
-T_{i}^{\mathrm{s}} & 0_{k}
\end{array}\right),\left(\begin{array}{cc}
0_{k} & 1_{k} \\
-1_{k} & 0_{k}
\end{array}\right)\right\} .
$$

By substituting (2.33) into (2.32) we are led to search for the set of $2 k \times 2 k$ real matrices $S$ and $A$ which commute with $\mathcal{T}$ for arbitrary values of the fields $W_{i}^{\mu}$ and $B^{\mu}$. It is sufficient to verify that this property holds for all elements of the set $\widehat{\mathcal{T}}$. Let

$$
X=\left(\begin{array}{ll}
X_{11} & X_{12} \\
X_{21} & X_{22}
\end{array}\right), \quad X=S, \quad \text { or } \quad X=A .
$$

Requiring commutativity [see eqs. (2.32) and (2.33)] with the three types of matrices in $\widehat{\mathcal{T}}$ we obtain the conditions

$$
\begin{aligned}
& X_{j k} T_{i}^{\mathrm{a}}=T_{i}^{\mathrm{a}} X_{j k}, \\
& X_{11} T_{i}^{\mathrm{s}}=T_{i}^{\mathrm{s}} X_{22}, \quad X_{22} T_{i}^{\mathrm{s}}=T_{i}^{\mathrm{s}} X_{11}, \quad X_{12} T_{i}^{\mathrm{s}}=-T_{i}^{\mathrm{s}} X_{21}, \quad X_{21} T_{i}^{\mathrm{s}}=-T_{i}^{\mathrm{s}} X_{12}, \\
& X_{11}=X_{22}, \quad X_{12}=-X_{21} .
\end{aligned}
$$

Using (2.36c) we find that $X_{11}$ and $X_{12}$ must commute with all matrices $T_{i}^{\mathrm{a}}, T_{i}^{\mathrm{s}}$ (assumed to form an irreducible representation). By Schur's lemma they must then be proportional to the $k \times k$ unit matrix, so that $S \propto \mathcal{I}$ and $A \propto \mathcal{J}$. Thus, the Lie algebra of the symmetry group of $K_{0}$ and $K_{1}$ consists of elements of the form

$$
L_{m n}=\mathcal{I} L_{m n}^{(\mathrm{a})}+\mathcal{J} L_{m n}^{(\mathrm{s})} .
$$

This is the Lie algebra of $\mathrm{U}(N)$ written in real variables.

The symmetries of $K_{0}$ and $K_{1}$ in the limit $\boldsymbol{g}^{\prime} \rightarrow 0$. A more interesting situation arises if we remove $\mathcal{J}$ from the set $\widehat{\mathcal{T}}$, as would apply to the limit $g^{\prime} \rightarrow 0$. Then we still find that $X_{11}+X_{22}$ and $X_{12}-X_{21}$ must commute with all matrices $T_{i}^{\mathrm{a}}, T_{i}^{\mathrm{s}}$, and hence must be proportional to the unit matrix. Further, $X_{11}-X_{22}$ and $X_{12}+X_{21}$ must commute with all $T_{i}^{\text {a }}$, but anticommute with all $T_{i}^{\mathrm{s}}$. For $k=2$, i.e. for the gauge group $\mathrm{SU}(2)_{L} \times \mathrm{U}(1)_{Y}$ in the limit $g^{\prime} \rightarrow 0$, we find that nonzero solutions of (2.32),

$$
X_{11}-X_{22} \propto \varepsilon \equiv \mathrm{i} \sigma^{2}, \quad X_{12}+X_{21} \propto \varepsilon,
$$

are possible [see eqs. (2.33) and (2.35)]. This means that the possible antisymmetric matrices $A$ may be any linear combination of matrices from the set

$$
\mathcal{G}=\left\{\left(\begin{array}{ll}
0 & \varepsilon \\
\varepsilon & 0
\end{array}\right),\left(\begin{array}{cc}
\varepsilon & 0 \\
0 & -\varepsilon
\end{array}\right), \mathcal{J}\right\}
$$

\footnotetext{
${ }^{7}$ Without the restriction $(2.32)$ there would be $\frac{1}{2} k(2 k+1) N(N-1)+\frac{1}{2} k(2 k-1) N(N+1)=k N(2 k N-1)$ independent terms, equal to the number of generators of $\mathrm{SO}(2 k N)$.
} 
where the $2 \times 2$ matrix $\varepsilon$ was defined in eq. (2.38). The set $\mathcal{G}$ is a basis of generators for $\mathrm{SU}(2)$. Thus, the Lie algebra of symmetry generators for $K_{0}$ and $K_{1}$ in this case consists of elements of the form

$$
L_{m n}=\mathcal{I} L_{m n}^{(\mathrm{a})}+\sum_{A \in \mathcal{G}} A L_{m n}^{(\mathrm{s})},
$$

allowing all possible symmetric $N \times N$ matrices $L^{(\mathrm{s})}$ for each $A$. There are $\frac{1}{2} N(N-1)+$ $\frac{3}{2} N(N+1)=2 N^{2}+N$ independent terms, equal to the number of generators of the $N \times N$ quaternionic symplectic group $\operatorname{Sp}(N)$. The generators (2.40) generate $\operatorname{Sp}(N)$, where the elements of $\mathcal{G}$ act as the quaternions $i, j$ and $k$.

The above results were again found under the assumption that the fields $W_{i}^{\mu}$ are arbitrary, and kept constant under the transformation. Combined $\mathrm{SU}(k)$ transformations of the $W_{i}^{\mu}$ and the $\Phi_{m}$ fields still remain a symmetry. This symmetry is enlarged to $\mathrm{SU}(k) \times \mathrm{Sp}(N)$ as $g^{\prime} \rightarrow 0$. (In the case $g^{\prime} \neq 0$ it is $\mathrm{SU}(k) \times \mathrm{U}(N)$.) In the case $k=2$ and $g^{\prime} \rightarrow 0$ the custodial $\mathrm{SO}(4)$ symmetry $^{8}$ is contained in $\mathrm{SU}(2) \times \mathrm{Sp}(N)$ in the following way: $\mathrm{SO}(4) \cong \mathrm{SU}(2)_{L} \times \mathrm{SU}(2)_{R} \subseteq \mathrm{SU}(2)_{L} \times \mathrm{Sp}(N) .{ }^{9}$ (More presicely, $\mathrm{SO}(4) \cong\left(\mathrm{SU}(2)_{L} \times\right.$ $\left.\mathrm{SU}(2)_{R}\right) / \mathbb{Z}_{2}$ [45].) The group $\mathrm{SU}(2)_{R} \subseteq \mathrm{Sp}(N)$ is generated (through exponentiation) by choosing $L^{(\mathrm{a})}=0_{N \times N}$ and each $L^{(\mathrm{s})} \propto I_{N \times N}$ in eq. (2.40). The generators of $\mathrm{SO}(4)$ are hence the 3 generators in $\mathcal{G}$ plus the 3 generators of the $\mathrm{SU}(2)_{L}$ gauge group (written in real form). Finally, the $\mathrm{U}(1)_{Y}$ hypercharge symmetry group is contained in $\mathrm{SU}(2)_{R}[25]$.

In the more general case of an $\mathrm{SU}(k) \times \mathrm{Sp}(N)$ symmetry of $K_{0}$ and $K_{1}$ in the limit $g^{\prime} \rightarrow 0$ there is, in the same manner as above, a "custodial" $\mathrm{SU}(k) \times \mathrm{SU}(2)_{R}$ symmetry, which also will contain $\mathrm{SU}(2) \times \mathrm{SU}(2)_{R} \cong \mathrm{SO}(4)$ subgroups.

The symmetries of $\boldsymbol{K}_{\mathbf{2}}$. Next, consider the terms quadratic in the gauge fields cf. eq. (2.29),

$$
K_{2}=-\sum_{n=1}^{N}\left(\Psi_{n}^{T}, \Theta_{n}^{T}\right) \mathcal{T}^{2}\left(\begin{array}{c}
\Psi_{n} \\
\Theta_{n}
\end{array}\right) .
$$

As in the symmetry analysis of $K_{1}$ we want to find all matrices $X$ such that $X \mathcal{T}^{2}=\mathcal{T}^{2} X$. All matrices $X$ which commute with $\widehat{\mathcal{T}}$ will fulfill this criterion (since $\mathcal{T}^{2}$ can be expanded in a set which consists of products of all possible pairs of matrices from $\widehat{\mathcal{T}}$ ). Therefore, the symmetries of $K_{1}$ are also symmetries of $K_{2}$.

\section{Spontaneous symmetry breakdown}

In this section we return to the case of $k=2$, i.e. with $\mathrm{SU}(2)_{L} \times \mathrm{U}(1)_{Y}$ as the gauge group and a row of $N$ Higgs doublets $\Phi_{m}$. Note however that many of our considerations have straightforward generalizations to $k>2$.

As for the Standard Model, the potential $V_{\mathrm{g}}$ of equation (2.5), or $V_{C}$ of equation (2.8), may acquire its minimum at nonzero values of the scalar fields, $\langle\Phi\rangle_{0}=\Phi^{(0)}$, where $\Phi$ (without a lower index) refers to the whole set of fields $\Phi_{m}$. This point, $\Phi^{(0)}$, will belong

\footnotetext{
${ }^{8}$ The custodial $\mathrm{SO}(4)$ symmetry cannot be extended to an $O(4)$ symmetry, see chapter 4 of [43].

${ }^{9}$ We are grateful to H. Haber, J. P. Silva and P. Ferreira for pointing out a mistake at this point in a previous manuscript.
} 
to one or more manifolds of equivalent minima related by the symmetries of the potential. One may use these symmetries to transform $\Phi^{(0)}$ to a particular form. A possible one is to require for $\Phi_{1}^{(0)}$ that only its lowest real component is nonzero. This can always be achieved by an $\mathrm{SU}(2)_{L} \times \mathrm{U}(1)_{Y}$ gauge transformation. Next, the upper component of $\Phi_{2}^{(0)}$ can be made real by the remaining $\mathrm{U}(1)$ gauge transformation which keeps $\Phi_{1}^{(0)}$ unchanged. Then one has no gauge freedom left to change $\Phi_{n}^{(0)}$ for $n \geq 3$. However, it was shown by Barroso et al. [31] that a sequence of unitary row transformations can shift the vacuum expectation values to the first two fields of the row only ${ }^{10}$ for instance (when written in complex form)

$$
\Phi_{1}^{(0)}=\left(\begin{array}{c}
0 \\
v_{1}
\end{array}\right), \quad \Phi_{2}^{(0)}=\left(\begin{array}{c}
u_{2} \\
v_{2} \mathrm{e}^{\mathrm{i} \delta}
\end{array}\right), \quad \Phi_{n}^{(0)}=0 \text { for } n \geq 3
$$

with $v_{1}, u_{2}, v_{2}$ and $\delta$ real. The special case $u_{2}=\delta=0$ is usually referred to as vacuum alignment, in which case we may also transform $v_{2}$ to zero by an orthogonal row transformation involving only $\Phi_{1}$ and $\Phi_{2}$. This is known as the Higgs basis [32-36]. However, for other purposes it may be more convenient to adopt a "democratic" basis in which the lower component of all (or most) fields $\Phi_{m}$ have a nonzero real expectation value. It is related to the Higgs basis by an orthogonal row transformation which preserves the form of $C$ and $\mathrm{U}(1)$ electromagnetic gauge transformations (the latter preserving the definition of electric charge).

Assume now the case of vacuum alignment and a potential $V_{O(4)}$ which is $O(4)$ invariant. Then the existence of the vacuum expectation values $\Phi^{(0)}$ will break the (explicit) symmetry down to $O(3)$, with the consequence that the Higgs boson particle spectra and other physical properties will organize themselves into multiplets of $O(3)$ (broken by perturbative corrections in $g^{\prime}$ ). The number of broken symmetry generators is 3 whether we consider the symmetry broken from $\mathrm{U}(2)$ to $\mathrm{U}(1)$ or from $O(4)$ to $O(3)$; this leads to the existence of 3 Higgs ghosts and no extra (pseudo-) Goldstone bosons. ${ }^{11}$

\subsection{Mass-squared matrices}

To make these statements slightly more explicit, as needed for calculation of the zero'th order (in $g$ and $g^{\prime}$ ) particle masses, we expand the potential around $\Phi^{(0)}$ to second order. There are no first order terms since we are expanding around a minimum. The matrix of second derivatives is the mass-squared matrix $M_{m n \alpha \beta}^{2}$. It is restricted by symmetries to have

\footnotetext{
${ }^{10}$ One may collect the quantities $\Phi_{m \alpha}^{(0)}(\alpha=1,2)$ into two $N$-component complex vectors $\tilde{\Phi}^{(1)}$ and $\tilde{\Phi}^{(2)}$. By a $\mathrm{U}(N)$ row transformation one may first rotate $\tilde{\Phi}_{m}^{(1)}$ so that only the component $\tilde{\Phi}_{1}^{(1)}$ is nonzero, with $\tilde{\Phi}_{1}^{(1)}$ real. There is a group of $\mathrm{U}(N-1)$ transformations preserving this condition; this may be used to transform $\tilde{\Phi}_{m}^{(2)}$ so that only the components $\tilde{\Phi}_{1}^{(2)}, \tilde{\Phi}_{2}^{(2)}$ are nonzero, with $\tilde{\Phi}_{2}^{(2)}$ real. One cannot do better due to the existence of four real $\mathrm{U}(N)$ invariant parameters in $\left\|\tilde{\Phi}^{(1)}\right\|,\left\|\tilde{\Phi}^{(2)}\right\|$, and $\tilde{\Phi}^{(1) \dagger} \tilde{\Phi}^{(2)}$. But there remains a $\mathrm{U}(N-2)$ group of transformations preserving this condition which can be used for other purposes. For an $\mathrm{SU}(k) \times \mathrm{U}(1)$ gauge group one may generalize this procedure to $k$ vectors $\tilde{\Phi}^{(j)}, j=1 \ldots k$.

${ }^{11}$ This remains true for general values of $k \geq 2$; a set of aligned vacuum expectation values will break $\mathrm{U}(k)$ to $\mathrm{U}(k-1)$ and $O(2 k)$ to $O(2 k-1)$. The number of broken generators is $2 k-1$ in both cases. The situation is different if we have two broken real directions, as in equation (3.1) with $u_{2}=0$ but $\delta \neq 0$. Cf. section 3.3 .
} 
a block diagonal form in the group indices $\alpha, \beta$. We use coordinates where $\Phi_{m}=\Psi_{m}+\mathrm{i} \Theta_{m}$ is expressed in terms of four real fields,

$$
\Phi_{m}=\Psi_{m}+\mathrm{i} \Theta_{m}=\left(\begin{array}{c}
\phi_{m 1}+\mathrm{i} \phi_{m 2} \\
v_{m}+\eta_{m}+\mathrm{i} \phi_{m 3}
\end{array}\right), \quad \Phi_{m}^{(0)}=\left(\begin{array}{c}
0 \\
v_{m}
\end{array}\right) .
$$

It is now convenient to represent these on real form as

$$
\Phi_{m}=\left(\begin{array}{c}
\Psi_{m} \\
\Theta_{m}
\end{array}\right)=\left(\begin{array}{c}
\phi_{m 1} \\
v_{m}+\eta_{m} \\
\phi_{m 2} \\
\phi_{m 3}
\end{array}\right) .
$$

We have the expansion

$$
V\left(\Phi^{(0)}+\Delta \Phi\right)=\langle V\rangle_{0}+\frac{1}{2}\left\langle\frac{\partial^{2} V}{\partial \Phi_{m \rho} \partial \Phi_{n \sigma}}\right\rangle_{0} \Delta \Phi_{m \rho} \Delta \Phi_{n \sigma}+\mathcal{O}\left(\Delta \Phi^{3}\right),
$$

where $\Phi_{m \rho}$ denotes one of the four possibilities $\phi_{m 1}, \eta_{m}, \phi_{m 2}, \phi_{m 3}$, and the subscript 0 indicates that a quantity is evaluated at $\Phi=\Phi^{(0)}$. Now a set of generators for $\mathrm{SO}(4)^{12}$ is

$$
\begin{array}{ll}
J_{1}=\left(\begin{array}{rrrr}
0 & 1 & 0 & 0 \\
-1 & 0 & 0 & 0 \\
0 & 0 & 0 & 0 \\
0 & 0 & 0 & 0
\end{array}\right), & J_{2}=\left(\begin{array}{rrrr}
0 & 0 & 0 & 0 \\
0 & 0 & -1 & 0 \\
0 & 1 & 0 & 0 \\
0 & 0 & 0 & 0
\end{array}\right), \quad J_{3}=\left(\begin{array}{rrrr}
0 & 0 & 0 & 0 \\
0 & 0 & 0 & -1 \\
0 & 0 & 0 & 0 \\
0 & 1 & 0 & 0
\end{array}\right), \\
J_{4}=\left(\begin{array}{rrrr}
0 & 0 & 0 & 0 \\
0 & 0 & 0 & 0 \\
0 & 0 & 0 & 1 \\
0 & 0 & -1 & 0
\end{array}\right), \quad J_{5}=\left(\begin{array}{rrrr}
0 & 0 & 0 & -1 \\
0 & 0 & 0 & 0 \\
0 & 0 & 0 & 0 \\
1 & 0 & 0 & 0
\end{array}\right), \quad J_{6}=\left(\begin{array}{rrrr}
0 & 0 & 0 & 0 \\
1 & 0 & 0 & 0 \\
0 & 0 & 0 & 0
\end{array}\right),
\end{array}
$$

where $J_{1}, J_{2}, J_{3}$ will transform the vacuum expectation value $\Phi^{(0)}$, while $J_{4}, J_{5}, J_{6}$ leave it unchanged, cf. eq. (3.3). In terms of these, the broken generators of the $\mathrm{SU}(2) \times \mathrm{U}(1)_{Y}$ gauge group, written in real form by the transformation $\rho$ defined in (B.1), are

$$
\frac{\mathrm{i}}{2} \sigma^{1} \rightarrow \frac{1}{2}\left(J_{2}+J_{5}\right), \quad \frac{\mathrm{i}}{2} \sigma^{2} \rightarrow \frac{1}{2}\left(J_{1}+J_{4}\right), \quad \frac{\mathrm{i}}{2}\left(1-\sigma^{3}\right) \rightarrow J_{3},
$$

and the unbroken $\mathrm{U}(1)$ (electromagnetic gauge) generator is

$$
\frac{\mathrm{i}}{2}\left(1+\sigma^{3}\right) \rightarrow J_{6}
$$

If now $V$ is invariant under

$$
\Delta \phi_{m 1} \rightarrow-\Delta \phi_{m 1}, \quad \Delta \eta_{m} \rightarrow \Delta \eta_{m}, \quad \Delta \phi_{m 2} \rightarrow-\Delta \phi_{m 2}, \quad \Delta \phi_{m 3} \rightarrow \Delta \phi_{m 3},
$$

there can be no terms in (3.4) mixing the sets $\left\{\Delta \phi_{1}, \Delta \phi_{2}\right\}$ and $\left\{\Delta \eta_{m}, \Delta \phi_{m 3}\right\}$. If $V$ in addition is invariant under

$$
\Delta \phi_{m 1} \rightarrow \Delta \phi_{m 2}, \quad \Delta \eta_{m} \rightarrow \Delta \eta_{m}, \quad \Delta \phi_{m 2} \rightarrow-\Delta \phi_{m 1}, \quad \Delta \phi_{m 3} \rightarrow \Delta \phi_{m 3},
$$

\footnotetext{
${ }^{12}$ Equivalently $O(4)$ : $\mathrm{SO}(4)$ and $O(4)$ have the same Lie algebra and hence the same generators.
} 
there can be no terms in (3.4) mixing $\Delta \phi_{m 1}$ and $\Delta \phi_{m 2}$, and we must have

$$
\left\langle\frac{\partial^{2} V}{\partial \phi_{m 1} \partial \phi_{n 1}}\right\rangle_{0}=\left\langle\frac{\partial^{2} V}{\partial \phi_{m 2} \partial \phi_{n 2}}\right\rangle_{0} \equiv M_{\mathrm{ch}, m n}^{2}
$$

We refer to this as the charged mass-squared matrix. The transformations considered generate a $\mathbb{Z}_{4}$ subgroup of the $\mathrm{U}(1)$ gauge group generated by $J_{6}$, assumed to be a symmetry of $V$. We have formulated it this way as a reminder that invariance under discrete subgroups may be sufficient to impose useful restrictions on the mass matrices.

If $V$ is invariant under $C$ transformations,

$$
\Delta \eta_{m} \rightarrow \Delta \eta_{m}, \quad \Delta \phi_{m 3} \rightarrow-\Delta \phi_{m 3}
$$

(irrespective of how we define $C$ to operate on the charged sector, e.g. $\Delta \phi_{m 1} \rightarrow \Delta \phi_{m 1}$, $\left.\Delta \phi_{m 2} \rightarrow-\Delta \phi_{m 2}\right)$ there can be no terms in (3.4) mixing $\Delta \eta_{m}$ and $\Delta \phi_{m 3}$. Thus the neutral mass-squared matrix decomposes into two more blocks, a $C$ even and a $C$ odd one,

$$
M_{C+, m n}^{2}=\left\langle\frac{\partial^{2} V}{\partial \eta_{m} \partial \eta_{n}}\right\rangle_{0}, \quad M_{C-, m n}^{2}=\left\langle\frac{\partial^{2} V}{\partial \phi_{m 3} \partial \phi_{n 3}}\right\rangle_{0} .
$$

If $V$ in addition is invariant under the transformations

$$
\Delta \phi_{m 1} \rightarrow \Delta \phi_{m 1}, \quad \Delta \eta_{m} \rightarrow \Delta \eta_{m}, \quad \Delta \phi_{m 2} \rightarrow-\Delta \phi_{m 3}, \quad \Delta \phi_{m 3} \rightarrow \Delta \phi_{m 2},
$$

which generate a $\mathbb{Z}_{4}$ subgroup of the $\mathrm{SO}(2)$ symmetry group generated by $J_{4}$, we obtain the relation

$$
M_{C-, m n}^{2}=M_{\mathrm{ch}, m n}^{2} .
$$

This explicitly demonstrates mass degeneracy between the charged and the $C$ odd sectors [37]. Especially, if the potential is $O(4)$-invariant (2.21), that is, we have a $C$-invariant theory where also the parameters $\lambda_{a b}^{(3)}=0$ [the latter implies (3.11)], ${ }^{13}$ the above symmetry criteria for mass degeneracy are valid. Moreover, since the renormalization is not changed when the Higgs fields acquire a vacuum expectation value [38-42], we do not get any mass renormalization counterterms from the quartic operators. So even though $O(4)$ violating quartic terms proportional to $\lambda_{a b}^{(3)}$ cannot alone be prohibited by any discrete symmetry imposed on the NHDM Lagrangian [43], they will not show up as counterterms when renormalizing the masses. Hence, the mass degeneration (3.12) will only be broken by loop corrections involving gauge bosons, since we get an exact $\mathrm{SO}(3)$ symmetry when $g^{\prime}=0$. With $g^{\prime} \neq 0$ and hence with an approximate $\mathrm{SO}(3)$ symmetry, the mass differences of the charged and $C P$-odd sectors will be of order $\mathcal{O}\left(g^{\prime 4}\right) \propto \mathcal{O}\left(e^{4}\right)$.

On the other hand, the $\mathrm{SO}(3)$ symmetry between $C P$-odd and charged sectors could also be broken by counterterms of the type $\lambda_{a b}^{(3)} \widehat{C}^{2}$, even though these terms are set to zero in the original potential, if we are considering scattering processes and not mass relations.

\footnotetext{
${ }^{13}$ For supersymmetric theories we typically have $\lambda_{a b}^{(3)} \neq 0$.
} 


\subsection{The Higgs ghosts}

Let $\Delta \Phi$ be chosen so that $\Phi^{(0)}+\epsilon \Delta \Phi+\mathcal{O}\left(\epsilon^{2}\right)$ is a family of minima related by the symmetry of the potential $V$,

$$
\frac{\partial}{\partial \Phi_{m \alpha}} V\left(\Phi^{(0)}+\epsilon \Delta \Phi\right)=0
$$

to first order in $\epsilon$. By differentiating this relation with respect to $\epsilon$ and then setting $\epsilon=0$ we find

$$
\left\langle\frac{\partial^{2} V}{\partial \Phi_{m \alpha} \partial \Phi_{n \beta}}\right\rangle_{0} \Delta \Phi_{n \beta}=0
$$

which reflects the fact that the matrix $M_{m n \alpha \beta}^{2}$ has zero eigenvalues with corresponding eigenvectors $\Delta \Phi_{n \beta}$. We may take the latter to be $\Delta \Phi^{(i)} \propto J_{i} \Phi^{(0)}$ for $i=1,2,3$. Normalized,

$$
\Delta \Phi_{m}^{(1)}=\left(v_{m}, 0,0,0\right)^{T} / a, \quad \Delta \Phi_{m}^{(2)}=\left(0,0, v_{m}, 0\right)^{T} / a, \quad \Delta \Phi_{m}^{(3)}=\left(0,0,0, v_{m}\right)^{T} / a,
$$

with $a^{2}=\sum_{m} v_{m}^{2}$. The massless excitations in these directions correspond to a triplet of Higgs ghosts. There will be $N-1$ additional $\mathrm{SO}(3)$ triplets of excitations in directions orthogonal to the ghosts. They correspond to physical particles. There will also be $N$ $\mathrm{SO}(3)$ singlets, transforming evenly under $C$, corresponding to physical particles. In the case of $N=2$, the triplet is $\left(H^{+}, H^{-}, A\right)$, whereas the singlets are $h$ and $H$ [18].

\subsection{Non-aligned vacuum expectation values}

We have assumed vacuum alignment in much of the previous discussions of this section. The phenomenologically most realistic deviation from this case is that we have a situation with two (real) broken directions, as in (3.1) with $u_{2}=0$ but $\delta \neq 0$. This corresponds to a situation which preserves the $\mathrm{U}(1)$ electromagnetic gauge symmetry, generated by $J_{6}$. Its corresponding definition of electric charge is preserved, but the $C$ symmetry (or, here equivalently, ${ }^{14} \mathrm{CP}$ symmetry) is spontaneously broken. In this situation, assuming

\footnotetext{
${ }^{14}$ For the Lagrangian (2.1), spontaneous $C$ and $C P$ violation (SCV and SCPV) are equivalent: By definition [44], $C P(C)$ is broken spontaneously if (1) There is a transformation that can be physically interpreted as $C P(C)$ and which keeps the Lagrangian invariant and (2) There is no transformation that can be physically interpreted as $C P(C)$ which keeps both the Lagrangian and the vacuum invariant.

$(S C V \Rightarrow S C P V)$. Assume $C$ is spontaneously broken, and implement $P$ by the spatial reflection

$$
\mathcal{P} \Phi_{n}(t, \boldsymbol{r}) \mathcal{P}^{\dagger}=\Phi_{n}(t,-\boldsymbol{r}) .
$$

Hence condition (1) of SCPV is satisfied. Next, assume condition (2) for SCPV is not satisfied. Then there is a $C P$ transformation

$$
(\mathcal{C P}) \Phi_{n}(t, \boldsymbol{r})(\mathcal{C P})^{\dagger}=U_{m n}^{C P} \Phi_{n}^{\dagger T}(t,-\boldsymbol{r}),
$$

which leaves both the Lagrangian and the vacuum unaltered. But then the $C$ transformation given by

$$
\mathcal{C} \Phi_{n}(t, \boldsymbol{r}) \mathcal{C}^{\dagger}=U_{m n}^{C} \Phi_{n}^{\dagger T}(t, \boldsymbol{r}),
$$

with $U_{m n}^{C}=U_{m n}^{C P}$ will infer that condition (2) of SCV does not hold, since the spatial reflection (3.16) does not change the vacuum nor the physics of the Lagrangian. This is a contradiction, and hence also condition (2) of SCPV must hold.

$(S C P V \Rightarrow S C V)$. Conversely, if $C P$ is spontaneously broken and condition (1) hence is satisfied by
}

$$
(\mathcal{C P}) \Phi_{n}(t, \boldsymbol{r})(\mathcal{C P})^{\dagger}=U_{m n}^{C P} \Phi_{n}^{\dagger T}(t,-\boldsymbol{r}),
$$


the potential only has the $\mathrm{SU}(2) \times \mathrm{U}(1)$ gauge symmetry, the three $\mathrm{SU}(2)$ generators are spontaneously broken, and we hence only get three Higgs ghosts and no (pseudo-) Goldstone bosons.

When we only have an $\mathrm{SU}(2) \times \mathrm{U}(1)$ symmetry in the potential, operators of the type $\widehat{C}$ are present in the quadratic or in the quartic part of the potential. Two of the excitations of the charged mass squared matrix are still massless, corresponding to the usual charged Higgs ghosts. ${ }^{15}$

In the case of non-aligned VEVs (or operators $\widehat{C}$ present in the quadratic part of the potential) the $C$ even and odd excitations generally mix to give a $2 N \times 2 N$ mass squared matrix for the neutral particles. Here one of the excitations will be massless, corresponding to a neutral Higgs ghost.

On the other hand, assume that $V$ is invariant under $O(4)$ transformations. Then there are no operators of the type $\widehat{C}$ present in the potential. The explicit $O(4)$ symmetry is now broken down to $O(2) \simeq \mathrm{U}(1)$, so that 5 generators are broken. As before, 3 of these will generate excitations which correspond to the Higgs ghosts; the remaining 2 will correspond to nearly massless charged pseudo-Goldstone ${ }^{16}$ bosons (massless to zero'th order in $g^{\prime}$ ).

To analyze the situation we again write $\Phi=\Phi^{(0)}+\Phi^{\prime}$ in terms of real fields,

$$
\Phi_{m}=\Phi_{m}^{(0)}+\left(\phi_{m 1}, \eta_{m}, \phi_{m 2}, \chi_{m}\right)^{T} \text { with } \Phi_{m}^{(0)}=\left(0, v_{m}, 0, w_{m}\right)^{T}
$$

$J_{4}$ and $J_{5}$ are now also broken by the vacuum expectation values. Acting with the broken generators on $\Phi^{(0)}$ one finds five eigenvectors of the mass matrix with zero eigenvalues,

$\overline{\text { we can re-implement the matrix } U^{C} \text { as } U^{C P}}$ above, and re-implement $P$ as the trivial transformation (3.16), and hence condition (1) of SCV is satisfied. Assume condition (2) of SCV does not hold. Then we, in a similar manner as for the case (SCV $\Rightarrow \mathrm{SCPV})$, can let $U^{C P}=U^{C}$ and define $P$ as in (3.16), and hence SCPV does not hold either, which is a contradiction. Hence condition (2) of SCV is satisfied.

${ }^{15}$ The relation between the complex and real formulations of the charged mass squared matrix is given by the map $\rho$ of appendix B: Let $M_{\mathrm{c}}^{2}$ denote the $N \times N$ complex mass squared matrix, and let $M_{\mathrm{r}}^{2}$ be the corresponding $2 N \times 2 N$ real matrix. They are related by

$$
\phi^{-\mathrm{T}} M_{\mathrm{c}}^{2} \phi^{+}=\left(\phi_{1} \phi_{2}\right) \rho\left(M_{\mathrm{c}}^{2}\right)\left(\begin{array}{l}
\phi_{1} \\
\phi_{2}
\end{array}\right),
$$

as can be seen from eq. (B.6). Then $\rho\left(M_{\mathrm{c}}^{2}\right)=M_{\mathrm{r}}^{2}$. The matrix $M_{\mathrm{c}}^{2}$ is Hermitian, and hence has only real eigenvalues. It then follows from the definition of $\rho$ that $\lambda$ is an eigenvalue of $M_{\mathrm{c}}^{2}$ if and only if $\lambda$ is an eigenvalue of $M_{\mathrm{r}}^{2}$. Moreover, if $v$ is an eigenvector of $M_{\mathrm{c}}^{2},(\operatorname{Re} v, \operatorname{Im} v)^{\mathrm{T}}$ and $(\operatorname{Im} v,-\operatorname{Re} v)^{\mathrm{T}}$ will be eigenvectors of $M_{\mathrm{r}}^{2}$ with the same eigenvalue $\lambda$. Hence oppositely charged particles will have the same mass, although terms $\phi_{1 m}$ and $\phi_{2 n}$ mix by the presence of operators $\widehat{C}_{m n}$ in the quadratic part of the potential or by complex (i.e. non-aligned) VEVs, and hence violate $C$. (This mass degeneration is a consequence of the remaining $\mathrm{U}(1) \cong \mathrm{SO}(2)$ hypercharge symmetry, i.e. that the generator $J_{6}$ is unbroken.) The identification (3.8) assumed that $\phi_{1 m}$ and $\phi_{2 n}$ did not mix, i.e. that $\operatorname{Im}\left(M_{\mathrm{c}}^{2}\right)=0$, cf. (B.1).

${ }^{16}$ Pseudo-Goldstone bosons stem from broken generators of the extra $O(4)$ symmetry of the potential, while Higgs ghosts by definition are generated by the broken generators of the gauge symmetry (which of course is a symmetry of the whole Lagrangian). The pseudo-Goldstone bosons acquire small masses from radiative corrections, and are hence not massless to all orders of perturbation theory, like true Goldstone bosons. True Goldstone bosons are, in contrast, generated by the spontaneous breaking of a symmetry of a total Lagrangian, not only a potential. 
$\Delta \Phi^{(i)} \propto J_{i} \Phi^{(0)}$. After normalization

$$
\begin{aligned}
& \Delta \Phi_{m}^{(1)}=\left(v_{m}, 0,0,0\right)^{T} / a, \\
& \Delta \Phi_{m}^{(2)}=\left(0,0,-v_{m}, 0\right)^{T} / a, \\
& \Delta \Phi_{m}^{(3)}=\left(0, w_{m}, 0,-v_{m}\right)^{T} / \sqrt{a^{2}+b^{2}} \\
& \Delta \Phi_{m}^{(4)}=\left(0,0, w_{m}, 0\right)^{T} / b, \\
& \Delta \Phi_{m}^{(5)}=\left(w_{m}, 0,0,0\right)^{T} / b,
\end{aligned}
$$

where $a^{2}=\sum_{m} v_{m}^{2}$ and $b^{2}=\sum_{m} w_{m}^{2}$. We see that all eigenvectors except $\Delta \Phi_{m}^{(3)}$ are in the charged sector, hence the two pseudo-Goldstone bosons (i.e. light Higgs bosons) mentioned above will be charged, as claimed. The eigenvectors in (3.18) are normalized, but they are not necessarily orthogonal to each other. Their nonvanishing inner products are

$$
\left(\Delta \Phi^{(1)}, \Delta \Phi^{(5)}\right)=-\left(\Delta \Phi^{(2)}, \Delta \Phi^{(4)}\right)=\frac{1}{a b} \sum_{m} v_{m} w_{m} \equiv \cos \vartheta .
$$

Here $|\sin \vartheta|>0$, since the vacuum expectation values by assumption are non-aligned. Thus, the orthonormalized eigenvectors corresponding to the Higgs ghosts can be written

$$
\begin{aligned}
& H_{m}^{(1)}=\frac{1}{\sqrt{a^{2}+b^{2}}}\left(v_{m}, 0, w_{m}, 0\right)^{T}=\frac{a}{\sqrt{a^{2}+b^{2}}} \Delta \Phi_{m}^{(1)}+\frac{b}{\sqrt{a^{2}+b^{2}}} \Delta \Phi_{m}^{(4)}, \\
& H_{m}^{(2)}=\frac{1}{\sqrt{a^{2}+b^{2}}}\left(w_{m}, 0,-v_{m}, 0\right)^{T}=\frac{a}{\sqrt{a^{2}+b^{2}}} \Delta \Phi_{m}^{(2)}+\frac{b}{\sqrt{a^{2}+b^{2}}} \Delta \Phi_{m}^{(5)}, \\
& H_{m}^{(3)}=\frac{1}{\sqrt{a^{2}+b^{2}}}\left(0, w_{m}, 0,-v_{m}\right)^{T}=\Delta \Phi_{m}^{(3)},
\end{aligned}
$$

where $H_{m}^{(i)} \propto G_{i} \Phi^{(0)}, G_{i}$ denoting the $\mathrm{SU}(2)$ generators as given by the map (3.6). The two eigenvectors corresponding to the Goldstone modes are orthogonal to those above,

$$
\begin{aligned}
& G^{(1)}=\frac{1}{\sin \vartheta \sqrt{a^{2}+b^{2}}}\left[a\left(\Delta \Phi^{(4)}+\cos \vartheta \Delta \Phi^{(2)}\right)-b\left(\Delta \Phi^{(1)}-\cos \vartheta \Delta \Phi^{(5)}\right)\right], \\
& G^{(2)}=\frac{1}{\sin \vartheta \sqrt{a^{2}+b^{2}}}\left[-a\left(\Delta \Phi^{(5)}-\cos \vartheta \Delta \Phi^{(1)}\right)+b\left(\Delta \Phi^{(2)}+\cos \vartheta \Delta \Phi^{(4)}\right)\right] .
\end{aligned}
$$

They have been orthonormalized. We note that the normalization constant becomes infinite in the limit of aligned vacuum expectation values, $\sin \vartheta \rightarrow 0$. We recall that the set $\left\{H^{(1)}, H^{(2)}, H^{(3)}, G^{(1)}, G^{(2)}\right\}$ are just numerical eigenvectors of the mass-squared matrix. The corresponding zero mode fields are the quantum fields obtained by projecting $\Phi^{\prime}$ on these eigenvectors,

$$
\Phi_{m \alpha}^{H^{(i)}}=\left(H^{(i)}, \Phi^{\prime}\right) H_{m \alpha}^{(i)}, \quad \Phi_{m \alpha}^{G^{(j)}}=\left(G^{(j)}, \Phi^{\prime}\right) G_{m \alpha}^{(j)} \quad \text { for } i=1,2,3 \text { and } j=1,2 .
$$

The field $\Phi^{H^{(3)}}$ is the neutral Higgs ghost field, while the fields $\Phi^{H^{(1)}}$ and $\Phi^{H^{(2)}}$ form the charged Higgs ghost field, and the fields $G^{(1)}$ and $G^{(2)}$ together form charged Goldstone boson fields.

If the vacuum expectation values broke the symmetry in even more directions, as in (3.1) with both $u_{2} \neq 0$ and $\delta \neq 0$, the situation would be different: All 6 generators of $\mathrm{SO}(4)$ would be broken, 4 of them corresponding to the 4 broken generators of the $\mathrm{U}(2)$ gauge group. Thus, there would be 2 pseudo-Goldstone bosons also in this case. 


\section{Concluding remarks}

We have analyzed the additional (approximate) symmetries which may arise in multi-Higgsdoublet models, due to the fact that the scalar potential may have more symmetries than required by the imposed gauge invariance. Moreover, for the kinetic terms we found that the symmetry group is $\mathrm{SU}(k) \times \mathrm{U}(N)$. In the limit $g^{\prime} \rightarrow 0$ the symmetry group of the kinetic terms is enhanced to $\mathrm{SU}(k) \times \mathrm{Sp}(N)$, which has an $\mathrm{SU}(k) \times \mathrm{SU}(2)$ subgroup. In the case $k=2$ the latter is the $\mathrm{SU}(2)_{L} \times \mathrm{SU}(2)_{R} \cong \mathrm{SO}(4)$ custodial symmetry. The most general $C$ invariant Higgs potential (2.8) has the same $\mathrm{SO}(4)$ symmetry, only broken by the presence of the operator $\widehat{C}^{2}$, that is, terms proportional to $\lambda_{a b}^{(3)}$. In the case where $\lambda_{a b}^{(3)}$ is set to zero, we have an exact mass degeneration (3.12) (assuming vacuum alignment) between charged and $C$ odd sectors in the limit $g^{\prime} \rightarrow 0$. When there is no vacuum alignment, but rather two broken (real) directions with the electromagnetic generator left unbroken, a pair of light, charged Higgs bosons emerge (cf. section 3.3).

The introduction of Yukawa couplings could further constrain the theory. With $N$ doublets, one could imagine "simplified" models analogous to Model I and Model II for the 2HDM, where only one doublet couples to all fermions, or where some doublets couple to up-type quarks, with others coupling to down-type fermions. Furthermore, with three or more doublets, one could arrange to let each fermion generation couple to its own doublet.

If $n$ doublets are without any Yukawa couplings, for example due to a discrete $\mathbb{Z}_{2}$ symmetry,

$$
\Phi_{i} \rightarrow-\Phi_{i}, \quad i=1, \ldots, n
$$

then such a sector would provide a dark matter candidate [8]. Indeed, with $n>1$, there would be a whole "family" of states in this "inert" sector, some of which would carry electric charge. Those would therefore be observable.

\section{A $P(k, \mathbb{R})$, the symmetry group of $\widehat{C}^{2}$}

We will here show that the set

$$
P(k, \mathbb{R})=\left\{S \in G L_{2 k}(\mathbb{R}) \mid S^{T} \mathcal{J} S= \pm \mathcal{J}\right\}
$$

given in eq. (2.17) is a Lie group: The associative law and the existence of the identity follow from $G L_{2 k}(\mathbb{R})$ (the set of all invertible, real $2 k \times 2 k$ matrices) being a group. Define

$$
P^{-}(k, \mathbb{R})=\left\{S \in G L_{2 k}(\mathbb{R}) \mid S^{T} \mathcal{J} S=-\mathcal{J}\right\}
$$

The other component of $P(k, \mathbb{R})$ (what we could call $P^{+}(k, \mathbb{R})$ ) is $\operatorname{Sp}(k, \mathbb{R})$. Then, if $S^{-} \in P^{-}$and $S^{+} \in \operatorname{Sp}(k, \mathbb{R})$, then we easily see by the definition that

$$
\begin{aligned}
& S^{-} S^{+}, S^{+} S^{-} \in P^{-}(k, \mathbb{R}), \\
& S_{1}^{+} S_{2}^{+}, S_{1}^{-} S_{2}^{-} \in \operatorname{Sp}(k, \mathbb{R}) .
\end{aligned}
$$


So the set $P(k, \mathbb{R})$ is closed under group multiplication. This set also includes the inverse of each element. We only have to show this for elements $S \in X^{-}$, since we already know $\operatorname{Sp}(k, \mathbb{R})$ is a Lie group. Let $S^{T} \mathcal{J} S=-\mathcal{J}$. Then

$$
\left(S^{T}\right)^{-1} S^{T} \mathcal{J} S S^{-1}=\left(S^{T}\right)^{-1}(-\mathcal{J}) S^{-1},
$$

and since we generally have that $\left(A^{T}\right)^{-1}=\left(A^{-1}\right)^{T}$,

$$
-\mathcal{J}=\left(S^{-1}\right)^{T} \mathcal{J} S^{-1},
$$

so $S^{-1} \in P^{-}$too (still, $P^{-}$is not a group considered isolated, since it is not closed under group multiplication, and does not include the identity).

We have now derived that $P(k, \mathbb{R})$ is a group. To prove it is a Lie group, we must prove that it is a (topologically) closed subset of $G L_{2 k}(\mathbb{R}): f(A)=A^{T} \mathcal{J} A$ is a continuous map, the set $\{ \pm \mathcal{J}\}$ is closed in $G L_{2 k}(\mathbb{R})$, and hence $P(k, \mathbb{R})=f^{-1}[\{ \pm \mathcal{J}\}]$ is closed in $G L_{2 k}(\mathbb{R})$.

The determinant of $\boldsymbol{P}^{-}(\boldsymbol{k}, \mathbb{R})$. We will now show that the determinant of the matrices in the set $P^{-}(k, \mathbb{R})$, consisting of the real matrices with the property $S^{T} \mathcal{J} S=-\mathcal{J}$, is $(-1)^{k}$ :

First, we claim the set $P^{-}(k, \mathbb{R})$ is given by

$$
P^{-}(k, \mathbb{R})=\operatorname{Sp}(k, \mathbb{R}) C=C \operatorname{Sp}(k, \mathbb{R}),
$$

with $C$ defined in eq. (2.13). This is so because if $S^{\prime} \in P^{-}(k, \mathbb{R})$, then $S^{\prime} C \in \operatorname{Sp}(k, \mathbb{R})$ since

$$
\left(S^{\prime} C\right)^{T} \mathcal{J}\left(S^{\prime} C\right)=C^{T}(-\mathcal{J}) C=\mathcal{J},
$$

and then $S^{\prime}=S C$ for $S=S^{\prime} C \in \operatorname{Sp}(k, \mathbb{R})$, since $C^{2}=I$. Similarly with $C \operatorname{Sp}(k, \mathbb{R})$.

On the other hand, if $S \in \operatorname{Sp}(k, \mathbb{R})$, then

$$
(S C)^{T} \mathcal{J}(S C)=C^{T} \mathcal{J} C=-\mathcal{J},
$$

so then $S C \in P^{-}(k, \mathbb{R})$. Similarly, $C S \in P^{-}(k, \mathbb{R})$.

Now we can evaluate the determinant of an arbitrary element in $S^{\prime} \in P^{-}(k, \mathbb{R})$. Since $S^{\prime}=S C$ for an element $S \in \operatorname{Sp}(k, \mathbb{R})$,

$$
\operatorname{det}\left(S^{\prime}\right)=\operatorname{det}(S) \operatorname{det}(C)=\operatorname{det}(C),
$$

since all matrices in $\operatorname{Sp}(k, \mathbb{R})$ have determinant 1 [46]. The determinant of an $n \times n$ matrix $A$ can be written (sum over repeated indices)

$$
\operatorname{det}(A)=\epsilon^{i_{1}, \ldots, i_{n}} A_{1, i_{1}} \cdots A_{n, i_{n}}
$$

(the Leibniz formula). Then there is only one non-zero term in this sum for the matrix $C$, so the determinant is given by (no sum over $k$ )

$$
\operatorname{det}(C)=\epsilon^{1,2, \ldots, 2 k} C_{1,1} C_{2,2} \cdots C_{2 k, 2 k}=1^{k}(-1)^{k}=(-1)^{k} .
$$

Hence by eqs. (A.9) and (A.11), the matrices of $P^{-}(k, \mathbb{R})$ have determinant $(-1)^{k}$. 
$\operatorname{Sp}(\boldsymbol{k}, \mathbb{R})$ and $\boldsymbol{P}^{-}(\boldsymbol{k}, \mathbb{R})$ are not connected. We want to show that $\operatorname{Sp}(k, \mathbb{R})$ and $P^{-}(k, \mathbb{R})$ are two components of $P(k, \mathbb{R})$, i.e. they are not connected. Connected means the same as path connected for Lie groups. Assume that the two components are connected. Then there has to be a continuous path between e.g. $I \in \operatorname{Sp}(k, \mathbb{R})$ and $C \in P^{-}(k, \mathbb{R})$. Let $X(t)$ be such a path, i.e. $X(0)=I$ and $X(1)=C$, where $X(t)$ is continuous. Consider the supremum

$$
t_{0}=\sup \left\{t \mid X^{T}(t) \mathcal{J} X(t)=+\mathcal{J}\right\} .
$$

We know that $X(1)^{T} \mathcal{J} X(1)=-\mathcal{J}$. Moreover, consider the function

$$
f(t)=\operatorname{det}\left(X^{T}(t) \mathcal{J} X(t)+\mathcal{J}\right),
$$

which is continuous for continuous functions $X(t)$, since the determinant, matrix addition, multiplication and transposition are continuous. But $f(t)$ is discontinuous for $t=t_{0}$, since there in any open interval containing $t_{0}$ will be values $t$ where $f(t)=0$ and other values where $f(t)=\operatorname{det}(2 \mathcal{J})=2^{2 k}$, by definition of $t_{0}$. Hence our assumption that $X(t)$ is continuous must be wrong, and hence the sets $\operatorname{Sp}(k, \mathbb{R})$ and $P^{-}(k, \mathbb{R})$ are not connected.

\section{B The map $\rho$}

We introduce a map $\rho$ which lets us easily translate between real and complex formulations of the kinetic terms we are studying. The map $\rho$ preserves both matrix multiplication, addition and the identity. ${ }^{17}$ We define $\rho$ as a function from $M_{k}(\mathbb{C})$, the set of all $k \times k$ complex matrices, to $M_{2 k}(\mathbb{R})$, the set of all $2 k \times 2 k$ real matrices by

$$
\rho(X)=\left(\begin{array}{cc}
\operatorname{Re}(X) & -\operatorname{Im}(X) \\
\operatorname{Im}(X) & \operatorname{Re}(X)
\end{array}\right) .
$$

With $U$ a Lie group, $\rho$ is a Lie group isomorphism from $U \subset M_{k}(\mathbb{C})$ to $\rho[U]$.

Now we want to show that the definition of $\rho$ can be extended to vectors so that it preserves products of complex vectors and matrices: Let $v$ be a complex $k \times 1$ vector, let $v=v_{R}+i v_{I}$, with $v_{R}, v_{I}$ real and define

$$
\rho(v) \equiv\left(\begin{array}{c}
\operatorname{Re}(v) \\
\operatorname{Im}(v)
\end{array}\right)=\left(\begin{array}{c}
v_{R} \\
v_{I}
\end{array}\right),
$$

and

$$
\rho\left(v^{\dagger}\right) \equiv\left(\operatorname{Re}\left(v^{\dagger}\right),-\operatorname{Im}\left(v^{\dagger}\right)\right)=\left(v_{R}^{T}, v_{I}^{T}\right) .
$$

Moreover, let $A$ be a complex $k \times k$ matrix and let $A=\left(A_{R}+i A_{I}\right)$, with $A_{R}, A_{I}$ real, then

$$
\rho(A v)=\rho(A) \rho(v)
$$

\footnotetext{
${ }^{17} \rho$ is an injective ring homomorphism [46]. On the other hand, the inclusion $\rho[\mathrm{U}(2)] \subset \mathrm{SO}(4)$ shows that $\rho$ does not preserve the determinant, even though it is a ring (or group) isomorphism on its image.
} 
since $\rho(A v)=\left(\begin{array}{c}(A v)_{R} \\ (A v)_{I}\end{array}\right)=\left(\begin{array}{cc}A_{R} & -A_{I} \\ A_{I} & A_{R}\end{array}\right)\left(\begin{array}{c}v_{R} \\ v_{I}\end{array}\right)=\rho(A) \rho(v)$. Furthermore, let $u, v$ be complex $k \times 1$ vectors, then

$$
\operatorname{Re}\left(u^{\dagger} A v\right)=\rho\left(u^{\dagger}\right) \rho(A) \rho(v)
$$

since $\operatorname{Re}\left(u^{\dagger} A v\right)=\operatorname{Re}\left[\left(u_{R}^{T}-i u_{I}^{T}\right)\left(A_{R}+i A_{I}\right)\left(v_{R}+i v_{I}\right)\right]=\left(\begin{array}{ll}u_{R}^{T} & u_{I}^{T}\end{array}\right)\left(\begin{array}{cc}A_{R} & -A_{I} \\ A_{I} & A_{R}\end{array}\right)\left(\begin{array}{l}v_{R} \\ v_{I}\end{array}\right)=$ $\rho\left(u^{\dagger}\right) \rho(A) \rho(v)$. Then,

$$
u^{\dagger} A v+v^{\dagger} A^{\dagger} u=\rho\left(u^{\dagger}\right) \rho(A) \rho(v)+\rho\left(v^{\dagger}\right) \rho\left(A^{\dagger}\right) \rho(u),
$$

since the left hand side of eq. (B.6) equals its real part.

Open Access. This article is distributed under the terms of the Creative Commons Attribution Noncommercial License which permits any noncommercial use, distribution, and reproduction in any medium, provided the original author(s) and source are credited.

\section{References}

[1] F. Englert and R. Brout, Broken symmetry and the mass of gauge vector mesons, Phys. Rev. Lett. 13 (1964) 321 [SPIRES].

[2] P.W. Higgs, Broken symmetries and the masses of gauge bosons, Phys. Rev. Lett. 13 (1964) 508 [SPIRES].

[3] G.S. Guralnik, C.R. Hagen and T.W.B. Kibble, Global conservation laws and massless particles, Phys. Rev. Lett. 13 (1964) 585 [SPIRES].

[4] P.W. Higgs, Spontaneous symmetry breakdown without massless bosons, Phys. Rev. 145 (1966) 1156 [SPIRES].

[5] V. Silveira and A. Zee, Scalar phantoms, Phys. Lett. B 161 (1985) 136 [SPIRES].

[6] D.E. Holz and A. Zee, Collisional dark matter and scalar phantoms, Phys. Lett. B 517 (2001) 239 [hep-ph/0105284] [SPIRES].

[7] E. Ma, Verifiable radiative seesaw mechanism of neutrino mass and dark matter, Phys. Rev. D 73 (2006) 077301 [hep-ph/0601225] [SPIRES].

[8] R. Barbieri, L.J. Hall and V.S. Rychkov, Improved naturalness with a heavy Higgs: An alternative road to LHC physics, Phys. Rev. D 74 (2006) 015007 [hep-ph/0603188] [SPIRES].

[9] B. Patt and F. Wilczek, Higgs-field portal into hidden sectors, hep-ph/0605188 [SPIRES].

[10] R.A. Porto and A. Zee, The private Higgs, Phys. Lett. B 666 (2008) 491 [arXiv:0712.0448] [SPIRES].

[11] B. Grzadkowski, O.M. Ogreid and P. Osland, Natural multi-Higgs model with dark matter and CP-violation, Phys. Rev. D 80 (2009) 055013 [arXiv:0904.2173] [SPIRES].

[12] T.D. Lee, A theory of spontaneous T violation, Phys. Rev. D 8 (1973) 1226 [SPIRES].

[13] J.F. Gunion, Extended Higgs sectors, in the proceedings of the 10th International Conference on Supersymmetry and Unification of Fundamental Interactions (SUSY02), Hamburg Germany, 17-23 June 2002 [hep-ph/0212150] [SPIRES]. 
[14] A. Wahab El Kaffas, P. Osland and O.M. Ogreid, Constraining the two-Higgs-doublet-model parameter space, Phys. Rev. D 76 (2007) 095001 [arXiv:0706.2997] [SPIRES].

[15] W. Grimus, L. Lavoura, O.M. Ogreid and P. Osland, A precision constraint on multi-Higgs-doublet models, J. Phys. G 35 (2008) 075001 [arXiv:0711.4022] [SPIRES].

[16] W. Grimus, L. Lavoura, O.M. Ogreid and P. Osland, The oblique parameters in multi-Higgs-doublet models, Nucl. Phys. B 801 (2008) 81 [arXiv: 0802.4353] [SPIRES].

[17] GFitTer collaboration, J. Haller, Fits of the electroweak standard model and beyond using Gfitter, arXiv:0810.3664 [SPIRES].

[18] J.F. Gunion, H.E. Haber, G.L. Kane and S. Dawson, The Higgs hunter's guide, Addison-Wesley, Reading U.K. (1990).

[19] S. Davidson and H.E. Haber, Basis-independent methods for the two-Higgs-doublet model, Phys. Rev. D 72 (2005) 035004 [Erratum-ibid. D 72 (2005) 099902] [hep-ph/0504050] [SPIRES].

[20] A. Barroso, P.M. Ferreira and R. Santos, Neutral minima in two-Higgs doublet models, Phys. Lett. B 652 (2007) 181 [hep-ph/0702098] [SPIRES].

[21] C.C. Nishi, The structure of potentials with $N$ Higgs doublets, Phys. Rev. D 76 (2007) 055013 [arXiv: 0706 . 2685] [SPIRES].

[22] H.E. Haber and A. Pomarol, Constraints from global symmetries on radiative corrections to the Higgs sector, Phys. Lett. B 302 (1993) 435 [hep-ph/9207267] [SPIRES].

[23] H.E. Haber and D. O'Neil, Basis-independent methods for the two-Higgs-doublet model III: The CP-conserving limit, custodial symmetry and the oblique parameters $S, T, U$, Phys. Rev. D 83 (2011) 055017 [arXiv: 1011.6188] [SPIRES].

[24] P. Sikivie, L. Susskind, M.B. Voloshin and V.I. Zakharov, Isospin breaking in technicolor models, Nucl. Phys. B 173 (1980) 189 [SPIRES].

[25] S. Willenbrock, Symmetries of the standard model, hep-ph/0410370 [SPIRES].

[26] S. Weinberg, Approximate symmetries and pseudoGoldstone bosons, Phys. Rev. Lett. 29 (1972) 1698 [SPIRES].

[27] S. Weinberg, Perturbative calculations of symmetry breaking, Phys. Rev. D 7 (1973) 2887 [SPIRES].

[28] R.A. Diaz, Phenomenological analysis of the two Higgs doublet model, hep-ph/0212237 [SPIRES].

[29] G.C. Branco, M.N. Rebelo and J.I. Silva-Marcos, CP-odd invariants in models with several Higgs doublets, Phys. Lett. B 614 (2005) 187 [hep-ph/0502118] [SPIRES].

[30] T. Cheng and L. Li, Gauge theory of elementary particle physics, Oxford University Press, Cambridge U.S.A. (1988).

[31] A. Barroso, P.M. Ferreira, R. Santos and J.P. Silva, Stability of the normal vacuum in multi-Higgs-doublet models, Phys. Rev. D 74 (2006) 085016 [hep-ph/0608282] [SPIRES].

[32] J.F. Donoghue and L.F. Li, Properties of charged Higgs bosons, Phys. Rev. D 19 (1979) 945 [SPIRES].

[33] H. Georgi and D.V. Nanopoulos, Suppression of flavor changing effects from neutral spinless meson exchange in gauge theories, Phys. Lett. B 82 (1979) 95 [SPIRES]. 
[34] L. Lavoura and J.P. Silva, Fundamental CP-violating quantities in a $\mathrm{SU}(2) \times \mathrm{U}(1)$ model with many Higgs doublets, Phys. Rev. D 50 (1994) 4619 [hep-ph/9404276] [SPIRES].

[35] L. Lavoura, Signatures of discrete symmetries in the scalar sector, Phys. Rev. D 50 (1994) 7089 [hep-ph/9405307] [SPIRES].

[36] F.J. Botella and J.P. Silva, Jarlskog-like invariants for theories with scalars and fermions, Phys. Rev. D 51 (1995) 3870 [hep-ph/9411288] [SPIRES].

[37] M.Aa. Solberg, Three-Higgs-doublet models, Master Thesis, University of Bergen, Bergen Norway (2004).

[38] B.W. Lee, Renormalization of the $\sigma$-model, Nucl. Phys. B 9 (1969) 649 [SPIRES].

[39] J.-L. Gervais and B.W. Lee, Renormalization of the $\sigma$-model (II) Fermion fields and regularization, Nucl. Phys. B 12 (1969) 627 [SPIRES].

[40] S. Weinberg, New approach to the renormalization group, Phys. Rev. D 8 (1973) 3497 [SPIRES].

[41] G. 't Hooft, Dimensional regularization and the renormalization group, Nucl. Phys. B 61 (1973) 455 [SPIRES].

[42] F. Cooper, B. Mihaila and J.F. Dawson, Renormalizing the Schwinger-Dyson equations in the auxiliary field formulation of $\lambda \phi^{4}$ field theory, Phys. Rev. D 70 (2004) 105008 [hep-ph/0407119] [SPIRES].

[43] M.Aa. Solberg, Dark matter candidates and their indirect detection, Ph.D. Thesis, NTNU, Trondheim Norway (2010)

[http://urn.kb.se/resolve?urn=urn:nbn:no:ntnu:diva-11162]

[44] G.C. Branco, L. Lavoura and J.P. Silva, CP violation, Int. Ser. Monogr. Phys. 103 (1999) 1.

[45] J.F. Cornwell, Group theory in physics. Vol. 2 (Techniques of Physics, 7), Academic, London U.K. (1984).

[46] A. Baker, Matrix groups, Springer, Berlin Germany (2002). 